\title{
Estimaciones de Respuesta al Ítem de los Parámetros de una Medida Breve de Bienestar Psicológico Estudiantil en el Contexto Universitario
}

\author{
Item response estimates of the parameters of a brief measure of student \\ psychological well-being in the university context
}

\author{
Josué Michael Franco Mendoza ${ }^{1}$ \\ Universidad Nacional Federico Villarreal
}

Recibido: $04-06-21$

Aceptado: $13-09-21$

Publicado: $23-12-21$

\section{Resumen}

El propósito del presente estudio fue el de construir una escala breve de bienestar psicológico en el contexto universitario, en fase preliminar. Para ello se contó con la participación de 417 estudiantes de la facultad de educación de una universidad pública de Lima, de ambos sexos, con edades oscilantes entre los 17 y 35 años $(M=23, D . S .=1.86)$. Se trabajó con los tópicos de Salud Mental (SM) y el de Familia y Relaciones Interpersonales (FR). El método fue instrumental, contando con una escala de 35 ítems y siete componentes, aprobada por cinco expertos a través del estadístico $V$ de Aiken, con 3 criterios de valoración $(V=.83,1.00)$. Los ítems pasaron por diferentes procesos de análisis en las estimaciones de parámetros con el modelo de dos parámetros logísticos (2PL) de la Teoría de Respuesta al Ítem (TRI), en contraste con indicadores de la Teoría Clásica de los Test (TCT), a partir del Análisis Factorial Confirmatorio (AFC) y medidas de consistencia interna. Los resultados evidenciaron que los componentes de la escala ajustaron adecuadamente al modelo de 2PL $\left(x^{2}=.58,1.73, p>\right.$ $.05)$, cumpliéndose los supuestos de unidimensionalidad e independencia local para los ítems por cada componente. Así también se reportaron funciones de información de los mismos, las cuales fueron complementadas con evidencias del ajuste de los AFC para cada tópico $\left(x^{2} / g l=1.18,2.45, G F I=.99,1.00, T L I=.94, .99, C F I=.95, .99, N N F I=.94, .99, R M R=\right.$ $.001, S R M R=.05, .06, R M S E A=.02, .06)$. Por otra parte, las medidas de consistencia interna oscilaron entre .61 y .82 , tomando como indicadores el coeficiente Omega y KR-20. Se recomienda continuar realizando revisiones del modelo, en una siguiente fase del estudio, a la vez de complementar análisis con componentes orientados a necesidades educativas y el desarrollo de habilidades blandas.

Palabras clave: Bienestar psicológico; construcción de pruebas; psicometría; estudiantes universitarios.

1 Estudiante de Psicología - Universidad Nacional Federico Villarreal, Lima, Perú. Autor para correspondencia: josuemovil7@gmail.com - 2015030558@unfv.edu.pe ORCID: https://orcid.org/0000-0003-3373-3334

(C) Los autores. Este artículo es publicado por la Revista de Investigación en Psicología de la Facultad de Psicología, Universidad Nacional Mayor de San Marcos. Este es un artículo de acceso abierto, distribuido bajo los términos de la licencia Creative Commons Atribución 4.0 Internacional (CC BY 4.0) [https://creativecommons.org/licenses/by/4.0/deed.es] que permite el uso, distribución y reproducción en cualquier medio, siempre que la obra original sea debidamente citada de su fuente original. 


\begin{abstract}
The purpose of the present study was to construct a brief scale of psychological well-being in the university context, in a preliminary phase. For this purpose, 417 students from the faculty of education of a public university in Lima, of both sexes, aged between 17 and 35 years $(M=$ 23, S.D. = 1.86), participated in the study. We worked with the topics of Mental Health $(\mathrm{MH})$ and Family and Interpersonal Relations (FR). The method was instrumental, with a scale of 35 items and seven components, approved by five experts through Aiken's V statistic, with three evaluation criteria $(V=.83,1.00)$. The items went through different analysis processes in parameter estimations with the two-logistic parameter model (2LP) of the Item Response Theory (IRT), in contrast with indicators of the Classical Test Theory (CTT), from the Confirmatory Factor Analysis (CFA) and internal consistency measures. The results showed that the scale components adequately adjusted to the $2 \mathrm{LP}$ model $\left(x^{2}=.58,1.73, p>.05\right)$, fulfilling the assumptions of unidimensionality and local independence for the items for each component. Information functions of the items were also reported, which were complemented with evidence of the CFA fit for each topic $\left(x^{2} / g l=1.18,2.45, G F I=.99,1.00, T L I=.94, .99\right.$, $C F I=.95, .99, N N F I=.94, .99, R M R=.001, S R M R=.05, .06, R M S E A=.02, .06)$. On the other hand, the internal consistency measures ranged between .61 and .82, taking the Omega coefficient and KR-20 as indicators. It is recommended to continue revising the model in a next phase of the study, while complementing the analysis with components oriented to educational needs and the development of soft skills.
\end{abstract}

Keywords: Psychological well-being; test construction; psychometrics; college students. 
Durante el desarrollo de la educación y docencia, se ha visto la continua necesidad por monitorear los aprendizajes de los estudiantes en distintos contextos que van desde la formación básica regular hasta la educación superior en institutos, universidades y múltiples centros laborales (Barrón-Estrada et al., 2018). Las maneras de llevar a cabo este proceso han ido evolucionando con diferentes estrategias y métodos que garanticen un adecuado desarrollo de las competencias y capacidades del estudiante, con base en modelos educativos que posibilitan la adopción del uso de herramientas o materiales físicos y digitales, desde que se tiene conocimiento por modelos de educación a distancia totales o parcializados (López, 2019; Reis et al., 2018).

En el marco de la coyuntura actual de la pandemia por COVID-19, son utilizadas cada vez más estrategias y recursos que busquen responder a las problemáticas que derivan de un sistema educativo de raíces presenciales, con la cobertura de la metodología de las modalidades de educación a distancia. Sin embargo, no todas las problemáticas dentro del contexto educativo son atendidas al mismo nivel, siendo uno de los principales ejes sin atender el de la tutoría y psicopedagogía, el cual entra dentro de las exigencias de muchas acreditadoras internacionales como también de las de la Superintendencia Nacional de Educación (SUNEDU), desde la promulgación de la nueva Ley Universitaria (Ley $\mathrm{N}^{\circ} 30220$ ), a partir de donde se estipula implementar servicios que brinden orientación en tres áreas importantes en la formación profesional del estudiantado: académica, social y personal, emergiendo estos, en el ámbito nacional privado, en modalidades presenciales o semi presenciales.

Sin embargo, no todas las necesidades estudiantiles a dichos niveles logran ser atendidas, en materias de igual o mayor importancia, como lo son los de su bienestar psicológico o emocional (Karlsen et al., 2006; Wright et al., 2013). Existen en el caso múltiples ejemplos que permiten verificar grandes casuísticas de problemas que suelen derivar de una gran crisis de salud mental en el país en la población estudiantil universitaria, como ha podido ser visto en los informes estadísticos del Instituto Nacional de Estadística e Informática ([INEI], 2018) o en investigaciones de corte internacional que nos retratan una multiplicidad de casos de violencia familiar, depresión, estrés, ansiedad, dependencia emocional, entre otros, que son detectados con mucha dificultad en el contexto académico universitario (Bahls, 2002; Hughes et al, 2010; Lemos y Londoño, 2006), si a esto también le sumamos la crisis cultural y falta de concientización de los adolescentes y jóvenes universitarios, en relación al cuidado de su salud mental (Washington, 2011; Castaños y Sánchez, 2015; Hudson \& Nandy, 2012). Siendo así, el impacto sobre miles de formandos a puertas de entrar al mercado laboral, puede verse potencial y funcionalmente comprometido, sobre todo, si es que los aspectos legalizados y procesos tradicionales no se ven totalmente abastecidos de condiciones de planeamiento continuo que respondan a la altura de las necesidades por atender dentro 
de este panorama de la realidad (Palummo, 2013). Es así que cabe destacar, más allá de la importancia que tiene lo académico, el papel de la salud psicológica de los estudiantes universitarios, considerando la influencia o el papel de diversos agentes socioculturales.

En distintos ámbitos de evaluación en salud mental, el bienestar psicológico es definido como el resultado de la calidad de vivencias de una persona en su espectro más amplio (Skin \& Johnson, 1978; Diener, 1996; García-Viniegras y González, 2000; Diener, Oishi \& Tay, 2018). En este sentido, dicho bienestar es trascendente en función de las experiencias que un individuo conserve con su entorno personal, social, familiar, escolar y cultural (Castellá et al., 2012). Implicancias en la amplitud del mismo concepto para el adolescente y joven adulto, pueden ser señaladas en las propuestas teóricas de Olivera y Simkin (2016), en relación a la conformación de factores hedónicos responsables de estructurar su personalidad; y así también, en los aportes de Rodríguez-Fernández et al. (2016), analizando este conjunto de vivencias, resaltando la importancia que toma el apoyo social percibido sobre el desarrollo de su resiliencia y manejo de emociones (Connor \& Davidson, 2003; Feitosa, de Matos y Del Prette, 2005; Chu et al., 2010) y el afecto que el joven encuentre dentro de su propio hogar, relación de pareja y entorno familiar (Lemos y Londoño, 2006; Pavot \& Diener, 2013). En el mismo sentido, durante el desarrollo de la formación del individuo, se hace de suma importancia el velar por la estabilidad de todos estos agentes, existiendo múltiples consecuencias negativas en función de la indulgencia en la atención a las necesidades emergentes, que puedan requerir acompañamiento, respuesta u orientación en su propio contexto (Crawford \& Manassis, 2011; Hughes et al, 2010).

Trayendo a lugar acotaciones más amplias, el bienestar psicológico del adolescente y joven adulto, en la vida universitaria, tiene también que ver con el desarrollo de una serie de habilidades que son de alto impacto para el resto de su desarrollo profesional. En este sentido, factores inter e intrapersonales, toman un papel clave de la respuesta que los estudiantes tendrán ante situaciones que los acompañarán desde el inicio hasta el desarrollo de una vida adulta más o menos saludable, siendo evidenciado en sus propios mecanismos de afrontamiento para la solución de sus problemas y toma de decisiones (Karlsen et al., 2006; VerdugoLucero et al., 2013; Fergus \& Zimmerman, 2005; Wright et al., 2013; Fuentes et al., 2012) en un gran número aspectos que lo acompañan en su proceso de adaptación a la comunidad profesional. (Gutiérrez y Gonçalves, 2013; Oliva et al., 2010; Pertegal et al., 2010; Lent et al.,2009). Con esto, podría decirse que el concepto del bienestar psicológico, en función de la literatura revisada, se puede sintetizar en 4 grandes tópicos, que logran acotar operacional y completamente este concepto: (1) Salud Mental, (2) Familia y relaciones interpersonales, (3) Necesidades Educativas y (4) Habilidades blandas o socioemocionales. Siendo así, se propone englobar, en amplio espectro, una sólida conceptualización del bienestar subjetivo 
o psicológico del estudiante universitario desde una síntesis que operacionaliza los diferentes tópicos anteriormente mencionados bajo las características que los componen, como se aprecia en la Tabla 1:

\section{Tabla 1}

Tópicos del Bienestar psicológico estudiantil

\begin{tabular}{ll}
\hline & \multicolumn{1}{c}{ Componentes } \\
\hline T1: Salud mental & - Ansiedad (Spielberg, 1966) \\
& - Depresión (Reynolds, s.f., en Ugarriza y Escurra, 2002) \\
& - Estrés (Chao et al., 2002) \\
& - Personalidad (Caprara et al., 1993) \\
& - Apoyo Social Percibido (Zimet et al., 1988) \\
T2: Familia y relaciones & - Estilos de crianza parental (APA, 2017) \\
interpersonales & - Situaciones de violencia familiar (Tenorio, 2011) \\
& - Dependencia Emocional (Lemos y Londoño, 2006) \\
T3: Necesidades educativas & - Estilos de aprendizaje (Felder y Silverman, 1988) \\
& - Estrategias de Aprendizaje (Beltrán, 1998; Meza, 2013) \\
& - Procrastinación académica (Busko, 1988; Trujillo-Chumán y Noé-Grijava, 2020) \\
& - Inteligencias múltiples (Gardner, 1995) \\
T4: Habilidades blandas & - Habilidades para la vida (Diaz et al., 2013) \\
\hline
\end{tabular}

A diferencia de los referentes teóricos anteriormente mencionados, se sintetiza, en una propuesta más parsimoniosa, la evaluación de diferentes componentes psicológicos que retratan las posibles situaciones de protección o riesgo sobre el bienestar estudiantil (Tenorio, 2011; Ugarriza y Escurra, 2002), la cual no solo comprende la dicotomía mencionada, sino también, el desarrollo de otras habilidades de utilidad para la vida y el ejercicio de su carrera o profesión (Diaz et al., 2013).

Es a partir de estas premisas que nace el Modelo de Medida Breve del Bienestar Psicológico que discrimina la situación de las personas evaluadas, en función de los 4 tópicos anteriormente mencionados, iniciando con 2 de los mismos: (1) Salud Mental y (2) Familia y Relaciones Interpersonales. De manera inicial, el modelo fue puesto a prueba a partir de la elaboración de 5 ítems por componente psicológico, para lo cual se empleó el modelo de respuesta al ítem (TRI) de 2 parámetros (López, 1995; Birnbaum, 1968; Baker, 1992), con la finalidad de estimar indicadores que evidencien adecuadas propiedades psicométricas en cada una de las escalas del modelo. Este mismo fue contrastado con los estimadores de la Teoría Clásica de los Test (TCT), con la finalidad de verificar la consistencia de la información proporcionada por las evidencias sobre el modelo propuesto (Ferrando y Anguiano, 2010; Muñiz y Fonseca-Pedrero, 2019). 


\section{MÉTODO}

\section{Diseño}

El estudio preliminar realizado fue instrumental (Montero y León, 2002), con medidas obtenidas en corte transversal, teniendo por finalidad el analizar las propiedades psicométricas de la medida breve de bienestar psicológico, a partir de estimaciones de parámetros de respuesta al ítem, bajo el contraste del modelo 2 parámetros logísticos con los de la Teoría Clásica de los Test (TCT).

\section{Participantes}

Bajo un muestreo no probabilístico, formaron parte del estudio un total de 417 estudiantes de una universidad pública de Lima metropolitana, con edades oscilantes entre los 17 y 35 años $(M=23, D . S .=1.86)$, de ambos sexos y distintos ciclos académicos, pertenecientes a la facultad de educación. Estos respondieron a los ítems de la escala, previo consentimiento informado, tomando en consideración las buenas prácticas éticas para el desarrollo de la investigación científica.

\section{Instrumentos}

Fue construida y empleada la Escala Breve de Bienestar Psicológico y aprobada con el dictamen de 5 expertos en la materia, a partir del estadístico V de Aiken, del cual se obtuvieron acuerdos consistentes entre los mismos. La escala fue construida con los componentes de Salud Mental: (1) Ansiedad estado, (2) Depresión, (3) Estrés y (4) Manejo emocional; a estos se sumaron 3 del tópico de Familia y Relaciones Interpersonales: (1) Apoyo Familiar Percibido, (2) Situaciones de Violencia Familiar y (3) Dependencia Emocional. Siendo así, quedó constituida una escala inicial de 35 ítems, que representaron 7 componentes del bienestar psicológico en 2 tópicos. Se decidió trabajar con este primer modelo breve, considerando la importancia y cantidad de elementos incluidos en la escala, previa revisión de la literatura.

\section{Procedimientos}

Se inició el estudio a partir de la construcción de la escala breve anteriormente mencionada, siendo evaluada por el juicio de cinco expertos, a través de tres criterios: relevancia, coherencia y claridad, con respuestas del grado de acuerdo de 1 a 3 puntos, del cual se esperó aprobación para dar continuidad al estudio. Cabe mencionar que los expertos consultados fueron psicólogos clínicos con más de cinco años de ejercicio de la profesión, que tomaron como base el paradigma cognitivo-conductual. Un paso posterior consistió en la redacción del consentimiento informado, explicando el propósito del estudio en el mismo documento previa evaluación de los participantes con la Escala. 
Es así, que se procedió a enviar ambos documentos en un formulario virtual para hacer recepción de las respuestas, de manera automatizada, sobre una hoja de cálculo cargada en la nube. Posteriormente, se procedió al análisis de los ítems, en función sus propiedades psicométricas con el modelo de dos parámetros logísticos, haciendo contraste entre indicadores de la TCT con los de la TRI, respetando los supuestos de ambos modelos. Así fueron realizados contrastes entre los modelos logísticos para evaluar cuál de ellos guardó mejores medidas de ajuste ante la propuesta planteada y estimaciones no paramétricas de dos modelos de ecuaciones estructurales, con medidas de confiabilidad reportadas, para el caso clásico.

\section{Análisis de datos}

Se empleó para el análisis de los datos el complemento eirt 2.0 (Yulianti et al., 2020), de descarga libre, en una hoja de cálculo, mediante el cual se obtuvieron medidas de TRI, con los modelos anteriormente especificados, tomando como medidas de ajuste al $x^{2}$. Los parámetros de la TCT fueron estimados, adicionalmente, con el Software JASP 0.14.1, software libre para estadística, desarrollado por la Universidad de Ámsterdam (Sánchez-Villena, 2019; Goss-Sampson, 2019).

Una vez perfilados los paquetes estadísticos, se calculó el coeficiente $\mathrm{V}$ de Aiken como un indicador de validez basada en el contenido por juicio de expertos; y luego de obtener medidas consistentes de acuerdo, fueron procesados los datos teniendo en cuenta indicadores de localización del rasgo latente y discriminación, a partir de los cuales se obtuvieron las funciones de información de cada ítem en los componentes evaluados. Las medidas de error se estimaron con la inversa de las funciones de información de las curvas características de los ítems y de cada Test. Asimismo, se procedió al análisis confirmatorio de los modelos a partir del Modelamiento de Ecuaciones Estructurales (SEM) robusto, con el estimador DWLS (Erceg-Hurn y Mirosevich, 2008; Ferrando y Anguiano, 2010). En el mismo sentido, fueron empleadas como medidas de confiabilidad coeficientes Omega y KR-20 para cada componente, desde la perspectiva de la TCT (Muñiz y Fonseca-Pedrero, 2019).

\section{RESULTADOS}

Se obtuvo respuesta del juicio de expertos a partir de la elaboración de la escala, siendo aprobatorias por cada componente, tomando como punto de corte el .80 para estimar el grado de acuerdo entre los expertos, a través de los criterios de relevancia, coherencia y claridad. Los resultados de este proceso fueron obtenidos mediante el estadístico $V$ de Aiken, del cual se muestran evidencias en la Tabla 2. 
Tabla 2

Validez de contenido para items en el tópico de Salud Mental

\begin{tabular}{lccccccc}
\hline \multirow{2}{*}{ Componente } & Ítems & \multicolumn{7}{c}{ V de Aiken (I.C. 95\%) } \\
\cline { 2 - 8 } & & \multicolumn{2}{c}{ Relevancia } & \multicolumn{2}{c}{ Coherencia } & \multicolumn{2}{c}{ Claridad } \\
\hline Ansiedad & 1 & 0.83 & $(0.49-0.96)$ & 0.83 & $(0.44-0.97)$ & 0.89 & $(0.52-0.98)$ \\
& 2 & 0.83 & $(0.49-0.96)$ & 0.83 & $(0.44-0.97)$ & 0.94 & $(0.65-0.99)$ \\
& 3 & 1.00 & $(0.68-1.00)$ & 1.00 & $(0.80-1.00)$ & 1.00 & $(0.85-1.00)$ \\
Depresión & 4 & 0.94 & $(0.39-1.00)$ & 1.00 & $(0.80-1.00)$ & 1.00 & $(0.85-1.00)$ \\
& 5 & 1.00 & $(0.68-1.00)$ & 0.83 & $(0.44-0.97)$ & 0.83 & $(0.44-0.97)$ \\
& 6 & 0.83 & $(0.49-0.96)$ & 0.89 & $(0.44-0.99)$ & 0.89 & $(0.44-0.99)$ \\
& 7 & 0.83 & $(0.49-0.96)$ & 0.83 & $(0.44-0.97)$ & 1.00 & $(0.68-1.00)$ \\
& 8 & 0.94 & $(0.39-1.00)$ & 0.89 & $(0.44-0.99)$ & 0.83 & $(0.44-0.97)$ \\
& 9 & 0.94 & $(0.39-1.00)$ & 1.00 & $(0.68-1.00)$ & 0.78 & $(0.49-0.93)$ \\
& 10 & 0.89 & $(0.34-0.99)$ & 0.89 & $(0.44-0.99)$ & 0.89 & $(0.52-0.98)$ \\
& 11 & 0.89 & $(0.34-0.99)$ & 0.89 & $(0.44-0.99)$ & 0.94 & $(0.65-0.99)$ \\
& 12 & 0.83 & $(0.49-0.96)$ & 0.89 & $(0.44-0.99)$ & 0.89 & $(0.52-0.98)$ \\
& 13 & 1.00 & $(0.68-1.00)$ & 1.00 & $(0.80-1.00)$ & 1.00 & $(0.85-1.00)$ \\
& 14 & 0.89 & $(0.34-0.99)$ & 0.89 & $(0.44-0.99)$ & 0.89 & $(0.52-0.98)$ \\
& 15 & 0.83 & $(0.49-0.96)$ & 0.83 & $(0.44-0.97)$ & 0.83 & $(0.48-0.96)$ \\
& 16 & 1.00 & $(0.68-1.00)$ & 1.00 & $(0.80-1.00)$ & 1.00 & $(0.85-1.00)$ \\
& 17 & 1.00 & $(0.68-1.00)$ & 1.00 & $(0.80-1.00)$ & 0.89 & $(0.52-0.98)$ \\
& 18 & 1.00 & $(0.68-1.00)$ & 1.00 & $(0.80-1.00)$ & 1.00 & $(0.85-1.00)$ \\
& 19 & 0.89 & $(0.52-0.98)$ & 0.83 & $(0.49-0.96)$ & 0.89 & $(0.44-0.99)$ \\
& 20 & 0.94 & $(0.65-0.99)$ & 0.94 & $(0.39-1.00)$ & 0.83 & $(0.44-0.97)$ \\
\hline
\end{tabular}

Para el tópico de Salud Mental (SM) se obtuvieron valores V de Aiken por encima de .80 , a partir de la valoración de los expertos, conforme los criterios de relevancia, coherencia y claridad de los ítems, situándose dentro de los valores esperados. El mismo procedimiento fue realizado para el tópico de Familia y Relaciones Interpersonales, tal y cual puede ser apreciado en la Tabla 3.

Del mismo modo, se obtuvieron valores consistentes, a partir del juicio de expertos para el segundo tópico de la escala breve, con medidas oscilantes entre .83 y 1. Estos resultados permitieron poner a prueba el modelo a partir de estimaciones de la TRI, contrastadas con las medidas de la TCT. En tal sentido, los resultados del proceso son presentados a partir de los modelos de 1 y 2 parámetros logísticos para los componentes de ambos tópicos como puede apreciarse desde la tabla 4 en adelante. Asimismo, se presentan las curvas características del test obtenidas para cada componente en las figuras 1 y 2 para los 417 estudiantes evaluados con la escala. 
Tabla 3

Validez de contenido para ítems en el tópico de Familia y Relaciones Interpersonales

\begin{tabular}{lccccccc}
\hline \multirow{2}{*}{ Componente } & Ítems & \multicolumn{7}{c}{ V de Aiken (I.C. 95\%) } \\
\cline { 2 - 7 } & \multicolumn{2}{c}{ Relevancia } & \multicolumn{2}{c}{ Coherencia } & \multicolumn{2}{c}{ Claridad } \\
\hline Apoyo Familiar & 21 & 1.00 & $(0.80-1.00)$ & 0.94 & $(0.39-1.00)$ & 0.89 & $(0.44-0.99)$ \\
& 22 & 1.00 & $(0.80-1.00)$ & 0.94 & $(0.39-1.00)$ & 1.00 & $(0.68-1.00)$ \\
& 23 & 0.94 & $(0.39-1.00)$ & 0.83 & $(0.49-0.96)$ & 0.83 & $(0.44-0.97)$ \\
Violencia Familiar & 24 & 0.89 & $(0.52-0.98)$ & 0.94 & $(0.39-1.00)$ & 0.89 & $(0.44-0.99)$ \\
& 25 & 0.89 & $(0.44-0.99)$ & 0.94 & $(0.65-0.99)$ & 1.00 & $(0.68-1.00)$ \\
& 27 & 0.89 & $(0.44-0.99)$ & 0.89 & $(0.52-0.98)$ & 0.89 & $(0.44-0.99)$ \\
Dependencia & 1.00 & $(0.80-1.00)$ & 1.00 & $(0.85-1.00)$ & 0.89 & $(0.44-0.99)$ \\
Emocional & 28 & 1.00 & $(0.80-1.00)$ & 1.00 & $(0.85-1.00)$ & 0.89 & $(0.44-0.99)$ \\
& 29 & 1.00 & $(0.80-1.00)$ & 0.89 & $(0.52-0.98)$ & 1.00 & $(0.80-1.00)$ \\
& 30 & 1.00 & $(0.80-1.00)$ & 0.94 & $(0.39-1.00)$ & 1.00 & $(0.80-1.00)$ \\
& 31 & 1.00 & $(0.80-1.00)$ & 1.00 & $(0.80-1.00)$ & 0.83 & $(0.44-0.97)$ \\
& 32 & 1.00 & $(0.68-1.00)$ & 0.83 & $(0.44-0.97)$ & 0.89 & $(0.44-0.99)$ \\
& 33 & 0.83 & $(0.49-0.96)$ & 0.89 & $(0.44-0.99)$ & 1.00 & $(0.80-1.00)$ \\
& 34 & 0.83 & $(0.49-0.96)$ & 0.83 & $(0.44-0.97)$ & 1.00 & $(0.80-1.00)$ \\
& 35 & 0.83 & $(0.44-0.97)$ & 0.89 & $(0.44-0.99)$ & 0.89 & $(0.52-0.98)$ \\
\hline
\end{tabular}

Se procesó inicialmente el análisis con el modelo de 2 parámetros logísticos (2PL) de la TRI, en donde se encontraron índices de ajuste adecuados al modelo por parte de todos los ítems, tanto para el tópico de salud mental como de familia y relaciones interpersonales $\left(x_{(\mathrm{SM})}^{2}=.58, x_{(\mathrm{FR})}^{2}=1.73, p>.05\right)$. En tal sentido, se procedió a analizar la independencia local de los ítems para cada tópico, comenzando por el de salud mental, cuyos resultados son presentados en la tabla 4.

Como puede apreciarse, la mayoría de los elementos se caracterizó por cumplir con el supuesto de independencia local, a excepción del ítem 2 y el 5 en la escala de depresión; sin embargo, esto no supone un problema en las medidas de ajustes obtenidas para el modelo planteado. El siguiente análisis consistió en las estimaciones de los parámetros logísticos del modelo, cuyos resultados son mostrados en la tabla 5.

A partir de la tabla pueden ser apreciados elementos con mayor y menor capacidad discriminativa como de posicionamiento sobre las funciones acumuladas que conforman las CCI para el modelo. Para el parámetro de discriminación (a) los índices oscilaron entre 1.04 y 2.40 para la escala de ansiedad, 1.05 y 2.38 para la de depresión, 1.72 y 4.21 para la de estrés, estando entre .31 y 1.84 la de manejo emocional. En cuanto a parámetros de localización (b) se obtuvieron índices oscilantes entre -.36 y .11 para la escala de ansiedad, -.51 y .50 para la de depresión, -1 y -.18 para la de estrés y -2.66 y -.95 para la de manejo emocional. 
Tabla 4

Pruebas de independencia local para items de Salud Mental

\begin{tabular}{|c|c|c|c|c|c|c|c|c|c|}
\hline \multicolumn{5}{|c|}{ Ansiedad } & \multicolumn{5}{|c|}{ Depresión } \\
\hline \multicolumn{2}{|c|}{ ítem-ítem } & \multirow{2}{*}{$\begin{array}{c}\mathbf{x}^{\mathbf{2}} \\
0.02\end{array}$} & \multirow{2}{*}{$\frac{\text { gl }}{1}$} & \multirow{2}{*}{$\begin{array}{c}\mathbf{P} \\
0.90\end{array}$} & \multicolumn{2}{|c|}{ ítem-ítem } & \multirow{2}{*}{$\begin{array}{c}\mathbf{x}^{\mathbf{2}} \\
1.93\end{array}$} & \multirow{2}{*}{$\begin{array}{c}\mathbf{g l} \\
1\end{array}$} & \multirow{2}{*}{$\frac{\mathbf{p}}{0.16}$} \\
\hline a1 & $\mathrm{a} 2$ & & & & $\mathrm{~d} 1$ & $\mathrm{~d} 2$ & & & \\
\hline al & $\mathrm{a} 3$ & 0.41 & 1 & 0.52 & $\mathrm{~d} 1$ & $\mathrm{~d} 3$ & 0.17 & 1 & 0.68 \\
\hline a1 & $\mathrm{a} 4$ & 0.05 & 1 & 0.82 & $\mathrm{~d} 1$ & $\mathrm{~d} 4$ & 1.03 & 1 & 0.31 \\
\hline al & a5 & 0.47 & 1 & 0.49 & $\mathrm{~d} 1$ & $\mathrm{~d} 5$ & 0.38 & 1 & 0.53 \\
\hline $\mathrm{a} 2$ & a3 & 0.39 & 1 & 0.53 & $\mathrm{~d} 2$ & $\mathrm{~d} 3$ & 0.05 & 1 & 0.82 \\
\hline $\mathrm{a} 2$ & $\mathrm{a} 4$ & 0.36 & 1 & 0.55 & $\mathrm{~d} 2$ & $\mathrm{~d} 4$ & 0.25 & 1 & 0.61 \\
\hline $\mathrm{a} 2$ & a5 & 0.67 & 1 & 0.41 & $\mathrm{~d} 2$ & $\mathrm{~d} 5$ & 4.48 & 1 & 0.03 \\
\hline a3 & $\mathrm{a} 4$ & 1.08 & 1 & 0.30 & $\mathrm{~d} 3$ & $\mathrm{~d} 4$ & 1.59 & 1 & 0.21 \\
\hline $\mathrm{a} 3$ & a5 & 0.98 & 1 & 0.32 & $\mathrm{~d} 3$ & $\mathrm{~d} 5$ & 0.55 & 1 & 0.46 \\
\hline $\mathrm{a} 4$ & a5 & 1.12 & 1 & 0.29 & $\mathrm{~d} 4$ & $\mathrm{~d} 5$ & 2.77 & 1 & 0.10 \\
\hline \multicolumn{5}{|c|}{ Estrés } & \multicolumn{5}{|c|}{ Manejo Emocional } \\
\hline \multicolumn{2}{|c|}{ ítem-ítem } & $\mathbf{x}^{2}$ & gl & $\mathbf{P}$ & \multicolumn{2}{|c|}{ ítem-ítem } & $\mathbf{x}^{2}$ & gl & $\mathbf{p}$ \\
\hline $\mathrm{e} 1$ & $\mathrm{e} 2$ & 0.10 & 1 & 0.75 & $\mathrm{~m} 1$ & $\mathrm{~m} 2$ & 0.32 & 1 & 0.57 \\
\hline el & e3 & 3.76 & 1 & 0.05 & $\mathrm{~m} 1$ & $\mathrm{~m} 3$ & 3.34 & 1 & 0.07 \\
\hline el & $\mathrm{e} 4$ & 0.06 & 1 & 0.80 & $\mathrm{~m} 1$ & $\mathrm{~m} 4$ & 0.02 & 1 & 0.88 \\
\hline el & e5 & 1.88 & 1 & 0.17 & $\mathrm{~m} 1$ & $\mathrm{~m} 5$ & 0.17 & 1 & 0.68 \\
\hline $\mathrm{e} 2$ & e3 & 0.18 & 1 & 0.67 & $\mathrm{~m} 2$ & $\mathrm{~m} 3$ & 0.53 & 1 & 0.47 \\
\hline $\mathrm{e} 2$ & $\mathrm{e} 4$ & 0.59 & 1 & 0.44 & $\mathrm{~m} 2$ & $\mathrm{~m} 4$ & 0.47 & 1 & 0.49 \\
\hline $\mathrm{e} 2$ & e5 & 0.25 & 1 & 0.62 & $\mathrm{~m} 2$ & $\mathrm{~m} 5$ & 0.00 & 1 & 0.98 \\
\hline e3 & $\mathrm{e} 4$ & 2.15 & 1 & 0.14 & $\mathrm{~m} 3$ & $\mathrm{~m} 4$ & 3.00 & 1 & 0.08 \\
\hline e3 & e5 & 0.21 & 1 & 0.65 & $\mathrm{~m} 3$ & $\mathrm{~m} 5$ & 1.33 & 1 & 0.25 \\
\hline e4 & e5 & 0.84 & 1 & 0.36 & $\mathrm{~m} 4$ & $\mathrm{~m} 5$ & 0.12 & 1 & 0.73 \\
\hline
\end{tabular}

Nota: Todos los ítems presentaron adecuado ajuste al modelo 2PL

Tabla 5

Parámetros de localización y discriminación para los items del tópico de Salud Mental

\begin{tabular}{lccccc}
\hline & Ítem & a & E.S. & b & E.S. \\
\hline Ansiedad & a1 & 2.60 & 0.25 & 0.12 & 0.05 \\
& a2 & 2.40 & 0.23 & 0.11 & 0.06 \\
& a3 & 1.04 & 0.13 & 0.25 & 0.11 \\
& a4 & 1.51 & 0.16 & -0.32 & 0.08 \\
Depresión & a5 & 1.22 & 0.14 & -0.36 & 0.10 \\
& d1 & 2.39 & 0.24 & -0.51 & 0.06 \\
& d2 & 1.17 & 0.14 & 0.16 & 0.10 \\
Estrés & d3 & 1.06 & 0.13 & -0.18 & 0.10 \\
& d4 & 1.58 & 0.16 & -0.02 & 0.07 \\
& d5 & 1.70 & 0.18 & 0.50 & 0.08 \\
& e1 & 2.02 & 0.20 & -0.41 & 0.07 \\
Manejo Emocional & e2 & 4.21 & 0.48 & -1.01 & 0.05 \\
& e3 & 2.19 & 0.22 & -0.39 & 0.06 \\
& e4 & 1.73 & 0.18 & -0.18 & 0.07 \\
& e5 & 4.05 & 0.46 & -1.01 & 0.05 \\
& m1 & 1.84 & 0.20 & -0.95 & 0.09 \\
\hline
\end{tabular}

Nota: E.S.: Error estándar 
En consecuencia, se formularon a partir de los parámetros las funciones de las CCT, comprendidas en el modelo. Dichas curvas son mostradas en la Figura 1, representando al test en su totalidad por cada componente $\mathrm{y}$, posteriormente, por cada ítem.

\section{Figura 1}

Curvas características de los Test y los items para el tópico de Salud Mental

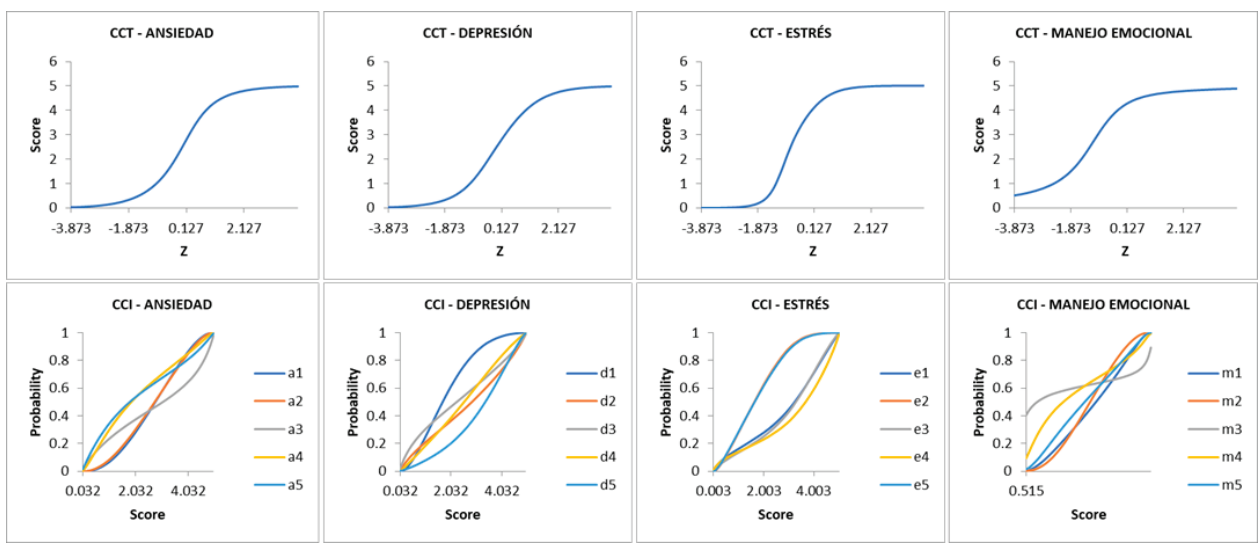

En tal sentido, las funciones de información del Test y los ítems quedaron conformadas a partir de los parámetros que definieron la capacidad discriminativa de cada elemento en cada escala. Dichas funciones son equivalentes a indicadores de consistencia interna o confiabilidad en la TCT. Los resultados obtenidos para estas funciones son mostrados en la Figura 2.

\section{Figura 2}

Funciones de Información de los Componentes en Salud Mental

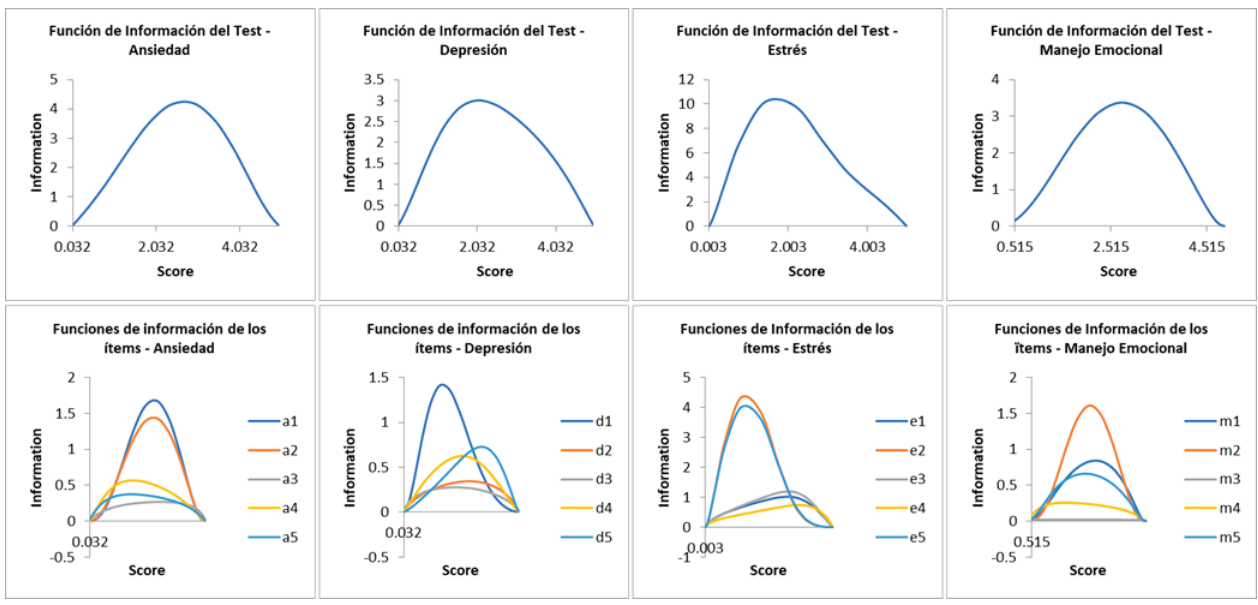


A partir de la figura, se hace notorio el funcionamiento diferencial de los ítems para detectar la presencia de cada componente psicológico en el tópico de salud mental; es decir, cada función de información ítem por ítem posee la capacidad de representar con mayor o menor notoriedad el rasgo medido de manera discriminante. Es así como, para la escala de ansiedad, los ítems 1 y 2 poseen más información que los ítems 4, 5 y 6; mientras que, en la escala de depresión, el ítem más representativo es el 1, frente a sus homólogos que se mantienen con un comportamiento similar. Del mismo modo, para la escala de estrés, los ítems 1 y 2 presentaron mayor capacidad informativa y para la escala de manejo emocional, el ítem 2. Los mismos procedimientos fueron realizados para los componentes de Familia y Relaciones Interpersonales.

Las medidas obtenidas de ajuste del modelo, basadas en el $x^{2}$ bondad de ajuste, evidenciaron medidas de ajuste adecuadas para el modelo de $2 \mathrm{PL}\left(x_{(\mathrm{SM})}^{2}=1.31\right.$, $\left.x^{2}{ }_{(\mathrm{FR})}=2.78, p>.05\right)$. A partir de estos hallazgos, fueron calculadas las medidas de los parámetros de los ítems en el modelo, poniendo a prueba de independencia local a los mismos, pese a no representar mayores problemas para la información ofrecida por los Tests en su totalidad, como en el caso anterior. Las medidas obtenidas se caracterizaron por cumplir, en la gran mayor parte de los casos, el supuesto previsto de independencia ( $p>.05)$, a excepción del ítem 2 de la escala de apoyo familiar, tal y como es mostrado de manera contigua en la tabla 6.

\section{Tabla 6}

Pruebas de independencia local para items de Familia y Relaciones Interpersonales

\begin{tabular}{|c|c|c|c|c|c|c|c|c|c|c|c|c|c|c|}
\hline \multicolumn{5}{|c|}{ Apoyo Familiar } & \multicolumn{5}{|c|}{ Violencia Familiar } & \multicolumn{5}{|c|}{ Dependencia Emocional } \\
\hline ítem & tem & $\mathbf{x}^{2}$ & gl & p & ítem & ítem & $\mathbf{x}^{2}$ & gl & p & íten & ítem & $\mathbf{x}^{2}$ & gl & $\mathbf{p}$ \\
\hline as1 & as2 & 0.09 & 1 & 0.77 & v1 & $\mathrm{v} 2$ & 0.07 & 1 & 0.79 & del & de2 & 0.67 & 1 & 0.41 \\
\hline as1 & as3 & 0.10 & 1 & 0.76 & vl & v3 & 0.72 & 1 & 0.40 & del & de 3 & 1.01 & 1 & 0.31 \\
\hline as1 & as4 & 0.24 & 1 & 0.62 & $\mathrm{vl}$ & v4 & 0.90 & 1 & 0.34 & del & de 4 & 0.19 & 1 & 0.66 \\
\hline as1 & as 5 & 0.02 & 1 & 0.90 & v1 & v5 & 0.06 & 1 & 0.80 & del & de 5 & 1.83 & 1 & 0.18 \\
\hline as2 & as 3 & 4.99 & 1 & 0.03 & $\mathrm{v} 2$ & v3 & 0.09 & 1 & 0.77 & $\operatorname{de} 2$ & de 3 & 1.83 & 1 & 0.18 \\
\hline as2 & as 4 & 0.01 & 1 & 0.93 & $\mathrm{v} 2$ & $\mathrm{v} 4$ & 0.34 & 1 & 0.56 & de2 & de 4 & 0.02 & 1 & 0.88 \\
\hline as2 & as5 & 0.04 & 1 & 0.85 & $\mathrm{v} 2$ & v5 & 0.58 & 1 & 0.45 & de2 & de 5 & 0.56 & 1 & 0.46 \\
\hline as3 & as 4 & 2.85 & 1 & 0.09 & v3 & $\mathrm{v} 4$ & 1.83 & 1 & 0.18 & de3 & de4 & 1.25 & 1 & 0.26 \\
\hline as 3 & as5 & 0.00 & 1 & 0.97 & v3 & v5 & 0.03 & 1 & 0.86 & de 3 & de 5 & 0.33 & 1 & 0.56 \\
\hline as4 & as5 & 0.48 & 1 & 0.49 & $\mathrm{v} 4$ & v5 & 0.50 & 1 & 0.48 & de 4 & $\operatorname{de} 5$ & 0.38 & 1 & 0.54 \\
\hline
\end{tabular}

Nota: Todos los ítems presentaron adecuado ajuste al modelo 2PL

En el mismo sentido, se presentan los parámetros de localización y discriminación, conforme el modelo de contraste elegido. Los resultados del proceso son mostrados en la Tabla 7, a partir de donde se evidencian distintas medidas que 
configuraron el funcionamiento diferencial de cada ítem. De manera consecuente, puede apreciarse cada curva característica, como en el caso anterior.

\section{Tabla 7}

Parámetros logísticos para los ítems del tópico de Familia y Relaciones Interpersonales

\begin{tabular}{|c|c|c|c|c|c|c|c|c|c|c|c|c|c|c|}
\hline \multicolumn{5}{|c|}{ Apoyo Familiar } & \multicolumn{5}{|c|}{ Violencia Familiar } & \multicolumn{5}{|c|}{ Dependencia Emocional } \\
\hline Ítem & $\mathbf{a}$ & E.S. & b & E.S. & Ítem & $\mathbf{a}$ & E.S. & b & E.S. & Ítem & $\mathbf{a}$ & E.S. & b & E.S. \\
\hline as1 & 3.20 & 0.44 & -1.63 & 0.09 & v1 & 2.28 & 0.24 & 0.96 & 0.08 & del & 1.16 & 0.15 & 1.08 & 0.14 \\
\hline as2 & 3.04 & 0.40 & -1.51 & 0.09 & v2 & 3.67 & 0.43 & 1.18 & 0.06 & de2 & 2.19 & 0.21 & 0.38 & 0.06 \\
\hline as3 & 0.55 & 0.12 & -1.91 & 0.42 & v3 & 2.85 & 0.33 & 1.19 & 0.07 & de3 & 2.70 & 0.30 & 1.01 & 0.07 \\
\hline as 4 & 2.31 & 0.27 & -1.30 & 0.09 & $\mathrm{v} 4$ & 2.10 & 0.21 & 0.63 & 0.07 & de4 & 1.20 & 0.15 & 1.11 & 0.14 \\
\hline as5 & 2.77 & 0.32 & -1.18 & 0.08 & v5 & 3.68 & 0.39 & 0.82 & 0.05 & de 5 & 2.28 & 0.24 & 0.81 & 0.07 \\
\hline
\end{tabular}

Los índices del parámetro de discriminación en cuanto al apoyo familiar oscilaron entre .55 y 3.20 , para los ítems de violencia familiar entre 2.10 y 3.68 , mientras que para dependencia emocional entre 1.16 y 2.70 . Asimismo, los parámetros de localización para el apoyo familiar se situaron entre -1.63 y -1.18; para los ítems de violencia familiar entre .63 y 1.19, situándose los de dependencia emocional entre .38 y 1.11. Dichas medidas representan proporcionalmente la capacidad de cada ítem para distinguir el rasgo latente de cada componente y las medidas de cada cual para representaciones de los mismos. De manera subsiguiente se presentan las CCT y CCI para el segundo tópico de la escala breve construida (ver Figura 3).

\section{Figura 3}

Curvas Características para Componentes en Familia y Relaciones Interpersonales

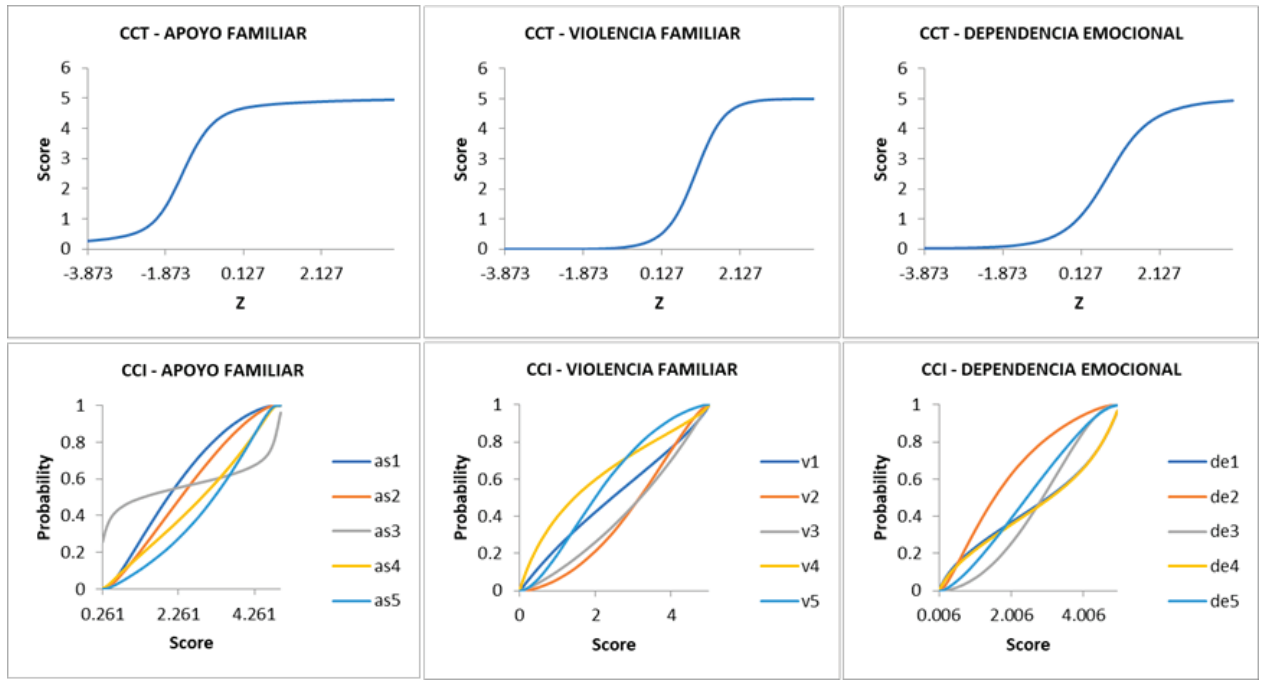


Como puede apreciarse, la mayo parte de los ítems se caracterizó por presentar un comportamiento diferencial entre pares. Por otro lado, es perceptible que se ubicó en una localización más negativa la escala de apoyo familiar, frente al restante de componentes.

En la Figura 4 puede ser apreciada la cantidad de información ofrecida por cada ítem para las escalas del segundo tópico del bienestar psicológico. El ítem más informativo para la escala de apoyo familiar fue el número 1 (21), mientras que para la escala de violencia familiar, los ítems 1 y 2 (26 y 27) y para la escala de dependencia emocional el ítem 3 (33). En tal sentido, es mostrado, gráficamente, el comportamiento de cada ítem, a partir de las funciones de información para el ítem y el Test, las cuales son inversas a las funciones de error para cada ítem y test.

Figura 4

Funciones de información para el tópico de Familia y Relaciones interpersonales

\begin{tabular}{|c|c|c|}
\hline 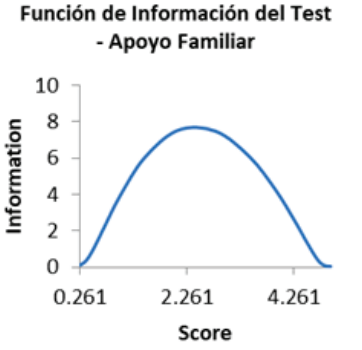 & 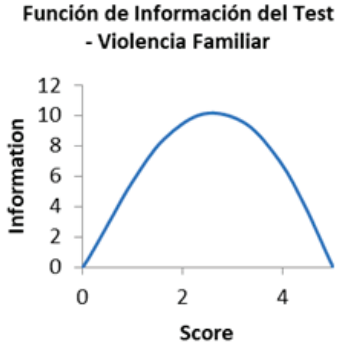 & $\begin{array}{c}\text { Función de Información del Test } \\
\text { - Dependencia Emocional }\end{array}$ \\
\hline $\begin{array}{l}\text { Funciones de Información de los } \\
\text { items - Apoyo Familiar }\end{array}$ & $\begin{array}{l}\text { Funciones de Información de los } \\
\text { ïtems - Violencia Familiar }\end{array}$ & $\begin{array}{l}\text { Función de Información de los } \\
\text { items - Dependencia Emocional }\end{array}$ \\
\hline
\end{tabular}

De manera consecuente, fueron analizadas medidas de la TCT para el modelo, a partir de las relaciones de concurrencia entre los componentes, haciendo uso de modelos de ecuaciones estructurales SEM (Structural Equiation Modeling), estimando medidas de ajuste del modelo para el Análisis Factorial Confirmatorio (AFC). En tal sentido, se presentan preliminarmente los índices obtenidos mediante el método robusto DWLS en la Tabla 8.

Se observaron índices de ajuste adecuados para los dos tópicos y 7 componentes unidimensionales de la escala breve de bienestar psicológico $\left(x^{2} / g l l_{(\mathrm{SM})}=\right.$ $\left.1.18, x^{2} / g l_{(\mathrm{FR})}=2.45, G F I_{(\mathrm{SM})}=.99, G F I_{(\mathrm{FR})} 1.00\right)$. Así también las medidas de los 
índices comparativos a la media se caracterizaron por ser superiores y situarse dentro de los parámetros esperados $\left(T L I_{(\mathrm{SM})}=.94, T L I_{(\mathrm{FR})}=.99, C F I_{(\mathrm{SM})}=.95, C F I\right.$ $\left.{ }_{(\mathrm{FR})}=.99, N N F I_{(\mathrm{SM})}=.94, N N F I_{(\mathrm{FR})}=.99\right)$, con escasos márgenes de error dentro de la estimación para cada modelo $\left(R M R_{(\mathrm{SM}, \mathrm{FR})}=.001, S R M R_{(\mathrm{SM})}=.05, S R M R_{(\mathrm{FR})}\right.$ $\left.=.06, R M S E A_{(\mathrm{SM})}=.02, R M S E A_{(\mathrm{FR})}=.06\right)_{\text {) }}$. En tal sentido se reportaron las cargas factoriales obtenidas, a partir de los ítems para cada componente, como se puede apreciar en las tablas 9 y 10.

\section{Tabla 8}

Estimaciones de ajuste del modelo factorial confirmatorio

\begin{tabular}{lcccccccc}
\hline & $\mathbf{x}^{2} / \mathbf{g l}$ & GFI & TLI & CFI & NNFI & RMSEA & SRMR & RMR \\
\hline Salud Mental (SM) & 2.45 & 1.00 & 0.94 & 0.95 & 0.94 & 0.06 & 0.06 & 0.01 \\
Fam. Y RI (FR) & 1.18 & 0.99 & 0.99 & 0.99 & 0.99 & 0.02 & 0.05 & 0.01 \\
\hline
\end{tabular}

Nota: $x_{(\mathrm{SM})}^{2}=401.448, g l_{(\mathrm{SM})}=164 ; x_{(\mathrm{FR})}^{2}=102.504, g l_{(\mathrm{FR})}=87$

\section{Tabla 9}

Cargas factoriales y covarianzas del modelo confirmatorio para Salud Mental

\begin{tabular}{|c|c|c|c|c|c|c|c|c|c|}
\hline & & \multirow[t]{2}{*}{ Ítem } & \multirow[t]{2}{*}{ Estimación } & \multirow[t]{2}{*}{ E.S. } & \multirow[t]{2}{*}{$\mathbf{Z}$} & \multirow[t]{2}{*}{ p } & \multicolumn{2}{|c|}{ IC (95\%) } & \multirow{2}{*}{$\begin{array}{c}\text { Carga } \\
(\lambda)\end{array}$} \\
\hline & & & & & & & Superior & Inferior & \\
\hline \multirow[t]{5}{*}{ AN } & $=\sim$ & al & 1.00 & 0.00 & & & 1.00 & 1.00 & 0.66 \\
\hline & $=\sim$ & $\mathrm{a} 2$ & 0.95 & 0.07 & 13.99 & $<.001$ & 0.81 & 1.08 & 0.62 \\
\hline & $=\sim$ & a3 & 0.89 & 0.06 & 13.85 & $<.001$ & 0.76 & 1.02 & 0.59 \\
\hline & $=\sim$ & $\mathrm{a} 4$ & 0.81 & 0.07 & 11.59 & $<.001$ & 0.68 & 0.95 & 0.54 \\
\hline & $=\sim$ & a5 & 0.63 & 0.08 & 8.40 & $<.001$ & 0.49 & 0.78 & 0.42 \\
\hline \multirow[t]{5}{*}{ DP } & $=\sim$ & $\mathrm{d} 1$ & 1.00 & 0.00 & & & 1.00 & 1.00 & 0.63 \\
\hline & $=\sim$ & $\mathrm{d} 2$ & 0.69 & 0.09 & 8.09 & $<.001$ & 0.53 & 0.86 & 0.42 \\
\hline & $=\sim$ & d3 & 1.00 & 0.08 & 12.33 & $<.001$ & 0.84 & 1.16 & 0.60 \\
\hline & $=\sim$ & $\mathrm{d} 4$ & 0.89 & 0.08 & 10.74 & $<.001$ & 0.73 & 1.05 & 0.53 \\
\hline & $=\sim$ & d5 & 0.75 & 0.08 & 9.91 & $<.001$ & 0.60 & 0.90 & 0.47 \\
\hline \multirow[t]{5}{*}{ ES } & $=\sim$ & el & 1.00 & 0.00 & & & 1.00 & 1.00 & 0.65 \\
\hline & $=\sim$ & e2 & 0.78 & 0.07 & 10.59 & $<.001$ & 0.64 & 0.93 & 0.65 \\
\hline & $=\sim$ & e3 & 1.05 & 0.07 & 14.93 & $<.001$ & 0.91 & 1.19 & 0.68 \\
\hline & $=\sim$ & e4 & 0.93 & 0.08 & 12.11 & $<.001$ & 0.78 & 1.09 & 0.59 \\
\hline & $=\sim$ & e5 & 0.80 & 0.08 & 10.17 & $<.001$ & 0.64 & 0.95 & 0.66 \\
\hline \multirow[t]{5}{*}{$\mathrm{ME}$} & $=\sim$ & $\mathrm{m} 1$ & 1.00 & 0.00 & & & 1.00 & 1.00 & 0.67 \\
\hline & $=\sim$ & $\mathrm{m} 2$ & 0.90 & 0.17 & 5.37 & $<.001$ & 0.57 & 1.22 & 0.66 \\
\hline & $=\sim$ & $\mathrm{m} 3$ & -0.13 & 0.12 & -1.10 & 0.27 & -0.37 & 0.10 & -0.08 \\
\hline & $=\sim$ & $\mathrm{m} 4$ & 0.25 & 0.12 & 2.08 & 0.04 & 0.01 & 0.48 & 0.18 \\
\hline & $=\sim$ & $\mathrm{m} 5$ & 0.64 & 0.15 & 4.17 & $<.001$ & 0.34 & 0.94 & 0.45 \\
\hline \multirow[t]{3}{*}{ AN } & $\sim \sim$ & DP & 0.09 & 0.01 & 11.61 & $<.001$ & 0.08 & 0.11 & 0.96 \\
\hline & $\sim \sim$ & ES & 0.08 & 0.01 & 10.57 & $<.001$ & 0.07 & 0.10 & 0.79 \\
\hline & $\sim \sim$ & $\mathrm{ME}$ & -0.04 & 0.01 & -4.67 & $<.001$ & -0.05 & -0.02 & -0.37 \\
\hline \multirow[t]{2}{*}{ DP } & $\sim \sim$ & ES & 0.07 & 0.01 & 8.90 & $<.001$ & 0.06 & 0.09 & 0.78 \\
\hline & $\sim \sim$ & $\mathrm{ME}$ & -0.04 & 0.01 & -4.91 & $<.001$ & -0.05 & -0.02 & -0.42 \\
\hline ES & $\sim \sim$ & ME & -0.02 & 0.01 & -3.09 & 0.002 & -0.03 & -0.01 & -0.23 \\
\hline
\end{tabular}


Fueron así observadas covarianzas directas fuertes entre la ansiedad con la depresión $(\lambda=.96)$ y el estrés $(\lambda=.79)$ e inversas con el manejo emocional $(\lambda=$ -.37), lo cual evidencia validez concurrente. Entre la depresión y el estrés se obtuvieron medidas de .78 y con el manejo emocional de -.42, siendo la relación inversa entre el estrés y el manejo emocional $(\lambda=-.23)$, siendo para todos los casos, muy significativas $(p<.01)$.

Del mismo modo se procedió al análisis de las cargas factoriales y covarianzas para los ítems de los componentes de Familia y Relaciones Interpersonales, pudiendo ser apreciados en la Tabla 10.

\section{Tabla 10}

Cargas factoriales y covarianzas del modelo confirmatorio para Familia y Relaciones Interpersonales

\begin{tabular}{|c|c|c|c|c|c|c|c|c|c|}
\hline & & \multirow[t]{2}{*}{ Ítem } & \multirow[t]{2}{*}{ Estimación } & \multirow[t]{2}{*}{ E.S. } & \multirow[t]{2}{*}{$\mathbf{Z}$} & \multirow[t]{2}{*}{$\mathbf{p}$} & \multicolumn{2}{|c|}{ IC (95\%) } & \multirow{2}{*}{ 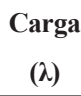 } \\
\hline & & & & & & & Superior & Inferior & \\
\hline \multirow[t]{5}{*}{$\mathrm{AF}$} & $=\sim$ & as1 & 1.00 & 0.00 & & & 1.00 & 1.00 & 0.60 \\
\hline & $=\sim$ & as2 & 1.34 & 0.26 & 5.24 & $<.001$ & 0.84 & 1.84 & 0.72 \\
\hline & $=\sim$ & as 3 & 0.32 & 0.19 & 1.69 & 0.091 & -0.05 & 0.70 & 0.12 \\
\hline & $=\sim$ & as4 & 1.31 & 0.27 & 4.91 & $<.001$ & 0.79 & 1.84 & 0.59 \\
\hline & $=\sim$ & as5 & 1.74 & 0.35 & 5.02 & $<.001$ & 1.06 & 2.42 & 0.76 \\
\hline \multirow[t]{5}{*}{ VF } & $=\sim$ & $\mathrm{v} 1$ & 1.00 & 0.00 & & & 1.00 & 1.00 & 0.65 \\
\hline & $=\sim$ & v2 & 0.90 & 0.09 & 9.80 & $<.001$ & 0.72 & 1.08 & 0.69 \\
\hline & $=\sim$ & v3 & 0.92 & 0.10 & 9.29 & $<.001$ & 0.73 & 1.11 & 0.69 \\
\hline & $=\sim$ & $\mathrm{v} 4$ & 1.05 & 0.10 & 10.83 & $<.001$ & 0.86 & 1.24 & 0.62 \\
\hline & $=\sim$ & v5 & 1.15 & 0.10 & 11.71 & $<.001$ & 0.96 & 1.34 & 0.74 \\
\hline \multirow[t]{5}{*}{$\mathrm{DE}$} & $=\sim$ & de1 & 1.00 & 0.00 & & & 1.00 & 1.00 & 0.49 \\
\hline & $=\sim$ & de2 & 1.28 & 0.20 & 6.36 & $<.001$ & 0.89 & 1.68 & 0.58 \\
\hline & $=\sim$ & de3 & 1.15 & 0.19 & 6.21 & $<.001$ & 0.79 & 1.51 & 0.63 \\
\hline & $=\sim$ & de4 & 0.87 & 0.16 & 5.54 & $<.001$ & 0.56 & 1.18 & 0.43 \\
\hline & $=\sim$ & de5 & 1.37 & 0.20 & 6.95 & $<.001$ & 0.98 & 1.75 & 0.68 \\
\hline \multirow[t]{2}{*}{$\mathrm{AF}$} & $\sim \sim$ & $\mathrm{DE}$ & 0.00 & 0.00 & -1.04 & 0.299 & -0.01 & 0.00 & -0.08 \\
\hline & $\sim \sim$ & $\mathrm{VF}$ & 0.07 & 0.01 & 6.50 & 0.002 & -0.03 & -0.01 & -0.34 \\
\hline VF & $\sim \sim$ & $\mathrm{DE}$ & 0.01 & 0.01 & 2.11 & 0.035 & $6.907 e-4$ & 0.02 & 0.16 \\
\hline
\end{tabular}

De la misma manera, se observaron cargas oscilantes entre .12 y .76, estando en proporciones adecuadas para con el ajuste del modelo. Las medidas de variabilidad entre componentes fueron directas entre la violencia familiar y la dependencia emocional $(\lambda=.16, p=.04)$, e inversas entre el apoyo y violencia familiar ( $\lambda$ $=-.34)$, como también, entre esta última con la dependencia emocional $(\lambda=-.08)$ 
siendo no significativas en este único caso. Siendo así, puede ser apreciada la distribución gráfica de los factores de ambos modelos a partir de la Figura 5.

Figura 5

Modelos Factoriales Confirmatorios en la Escala Breve de Bienestar Psicológico

Modelo 1. Salud Mental

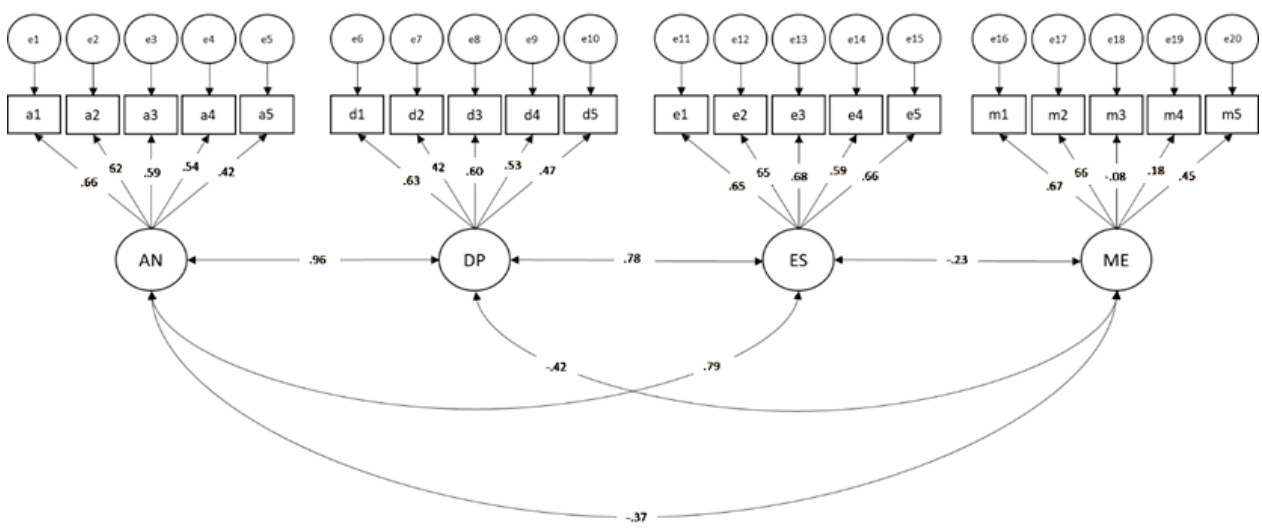

Modelo 2. Familia y Relaciones Interpersonales

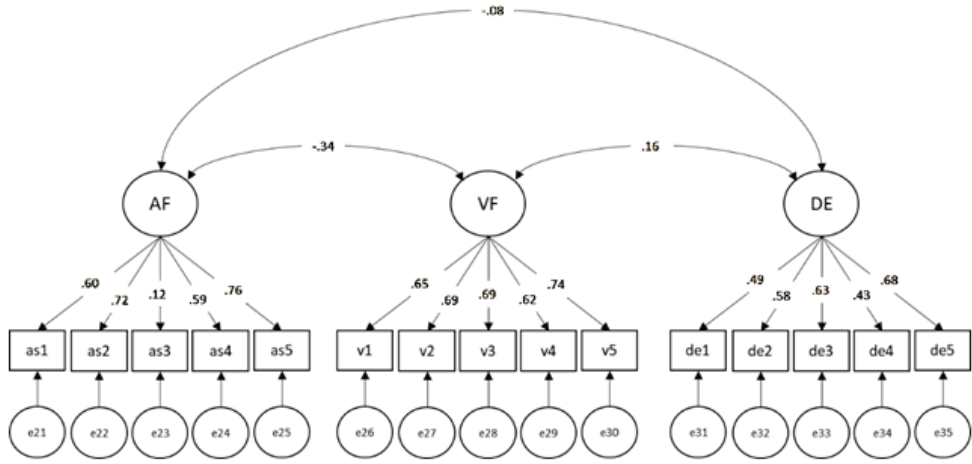

Con la serie de análisis realizados se confirman ambos modelos dentro de la Escala elaborada. En consecuencia, se llevaron a cabo análisis de la confiabilidad mediante el método de consistencia interna, por homogeneidad. Se obtuvieron así medidas consistentes emergentes de los modelos factoriales para ambos tópicos en cada componente, a partir de estimaciones del coeficiente Omega de McDonald y KR-20. Los resultados de dichas medidas son presentados en la Tabla 11.

Las medidas de consistencia obtenidas para el KR-20 oscilaron entre .61 y .81, entrando en un rango considerablemente adecuado, a pesar de la poca cantidad de elementos para cada componente de la escala. Del mismo modo, para el coeficiente 
Omega, los valores obtenidos se situaron entre .62 y .81 , refiriendo medidas adecuadas de confiabilidad, a partir de ambos indicadores.

Tabla 11

Medidas de confiabilidad por consistencia interna

\begin{tabular}{llccc}
\hline \multicolumn{1}{c}{ Tópico } & \multicolumn{1}{c}{ Componente } & No ítems & KR-20 & Omega \\
\hline Salud Mental & Ansiedad & 5 & 0.70 & 0.70 \\
& Depresión & 5 & 0.66 & 0.67 \\
& Estrés & 5 & 0.77 & 0.79 \\
& Manejo Emocional & 5 & 0.61 & 0.62 \\
Familia y RI & Apoyo Familiar & 5 & 0.66 & 0.68 \\
& Violencia Familiar & 5 & 0.81 & 0.81 \\
& Dependencia Emocional & 5 & 0.69 & 0.70 \\
\hline
\end{tabular}

En este amplio sentido, se evidenciaron adecuadas propiedades psicométricas para las medidas de la Escala Breve de Bienestar Psicológico, a partir de indicadores de la TRI y TCT, con los cuales se confirmó la presencia de factores que cumplieron una función diferencial por cada componente medido, desde los ítems que constituyeron ambos tópicos. Queda pendiente, por tanto, el evaluar las propiedades psicométricas de componentes adicionales, asociados a las necesidades educativas estudiantiles y el desarrollo de habilidades blandas o socioemocionales.

\section{DISCUSIÓN}

El objeto del presente estudio fue el de construir un modelo de medida breve del bienestar psicológico, en el contexto universitario. A partir de ello, se pusieron a prueba el modelo logístico de 2 parámetros de la TRI con el análisis SEM, en función de la TCT para dos tópicos de la propuesta teórica: (1) Salud Mental y Familia y (2) Relaciones Interpersonales, con evidencias en fase preliminar. Los resultados obtenidos evidenciaron un adecuado ajuste de la Escala con ambos modelos psicométricos, lo cual da por sentada la evidencia a favor del empleo y estudio de la misma y el modelo propuesto con mayor profundidad, permitiendo estos el facilitar la evaluación de situaciones de riesgo en el bienestar estudiantil universitario.

Las evidencias obtenidas, preliminarmente, del funcionamiento del modelo, contribuyen a diferentes aspectos metodológicos, dentro del uso de un instrumento de libre acceso para la comunidad psicológica y psicopedagógica en el contexto universitario, contándose con medidas adecuadas de validez y confiabilidad que extienden su valor hacia las experiencias sobre las cuales los estudiantes universitarios definen la vivencia y el sentido de bienestar psicológico (García-Viniegras y González, 2000). El sentido de la complejidad del concepto se hace contrastable con los alcances que han tenido otros modelos, tales como los de Castellá et al. 
(2012) u Olivera y Simkin (2016); haciéndose más concordante con la propuesta que redefine al individuo dentro de su espectro más amplio y completo (Oishi \& Tay, 2018; Skin \& Johnson, 1978; Diener, 1996).

En tal sentido, se hace notoria la presencia de múltiples agentes involucrados en el concepto de bienestar psicológico, pasando desde la perspectiva humanista, a indicadores cognitivo comportamentales, a partir de una amplia revisión de la literatura al respecto para redefinir el concepto en ámbitos de aplicación homologada. Es así que el modelo propuesto busca responder a las necesidades emergentes del contexto juvenil, enmarcado en motivos comunes y prevalentes consulta en el campo clínico, familiar, social, cultural y del desarrollo de habilidades u otras competencias socioemocionales ya validadas (Connor \& Davidson, 2003; Chu et al., 2010; Pavot \& Diener, 2013). Este primer proceso permitió distinguir qué elementos funcionan con mayor potencial discriminativo para el tamizaje de situaciones de mayor o menor riesgo en la población especificada, donde claramente la presencia de factores asociados a la salud mental y la familia cobran vigencia en el ámbito de desarrollo de los estudiantes evaluados (Castaños y Sánchez, 2015; Hudson \& Nandy, 2012; Connor \& Davidson, 2003).

Las limitaciones sobre este estudio se dieron a partir del alcance sobre la implementación de un gran conjunto de ítems que podrían representar al modelo, en toda su complejidad; sin embargo, este aspecto podría también representar situaciones de agotamiento para los estudiantes (Muñiz, 2019; Haladyna y Rodriguez, 2013), teniéndose en consecuencia puntuaciones menos precisas o validables, a partir de las estrategias empleadas (Muñiz y Bartram, 2007; Muñiz, 2019). Es por este motivo que se decidió dividir el estudio en diferentes fases, considerando que el desglose de los componentes de los demás tópicos, suman alrededor de $30 \mathrm{com}$ ponentes; y estos, a su vez, se subdividen en subcomponentes más específicos. Siguiendo la misma premisa para los componentes restantes de 5 ítems por cada uno, la medida breve podría la característica de perder el concepto de brevedad para una evaluación menos específica, siendo equivalente a tomar múltiples test bajo los modelos anteriormente señalados. Estas son unas de las razones principales por las cuales también se optó por el trabajar sobre componentes psicológicos, en función de un tamizaje de mayor potencial predictivo, respetando el concepto de los indicadores que se mencionaron para concebir cada componente, como referente teórico, bajo la mirada del modelo cognitivo conductual, en formato de autorreporte, donde las evidencias apuntan adecuadas funcionalidades del formato (Peñate et al., 2014; Fernández Castillo et al., 2014; Fernández, 2005).

Las medidas de confiabilidad obtenidas, con respaldo del AFC y los parámetros del modelo de 2PL de la TRI se adecuan a un modelo para el tamizaje, como una de las herramientas principales dentro del trabajo psicológico (Abad et al., 2006; Muñiz y Fonseca-Pedrero, 2019; Cuesta, 1996; Elosua y Mirosevich, 2008). Es así que se recomienda su uso, en casos donde la evaluación psicológica sea 
específica y estrictamente diferencial para cada criterio tópico. Bajo estos mismos alcances, se hace necesario continuar haciendo revisiones del instrumento con otros que cuenten con evidencias sólidas y publicitadas para la comunidad científica, para personas de diferentes localidades, dentro y fuera de la región (Elosua y Mirosevich 2008; Muñiz y Fonseca-Pedrero, 2019). Dentro de esta serie de análisis, cabe destacar la importancia de dar continuidad al estudio de la capacidad predictiva del modelo, componente a componente, para medir el bienestar psicológico del estudiante universitario, en función de los posibles riesgos señalados.

Finalmente, es preciso señalar que se dará continuidad al estudio del modelo, incluyendo los componentes de los tópicos de Necesidades Educativas y Habilidades Blandas, mejorando los procesos de validación anteriormente empleados. Así también, se da carta abierta al estudio del modelo propuesto, en función de las evidencias encontradas para brindar mayores soportes empíricos, en la práctica de la psicología y psicopedagogía universitaria.

\section{CONCLUSIONES}

La Escala Breve de Bienestar Psicológico cuenta con adecuadas propiedades psicométricas, ajustándose al modelo de 2 parámetros logísticos de la TRI y los modelos SEM de la TCT. Para el corto número de ítems por componente psicológico, las evidencias a favor de la escala son sólidas y presentan relaciones de convergencia entre constructos.

El estudio debe ser continuado con el resto de componentes en los tópicos de Necesidades Educativas y Habilidades Blandas. Estos deben ser llevados a cabo con mejoras en la implementación metodológica para profundizar en el análisis de sus estudios.

\section{Agradecimientos / Acknowledgments:}

Se agradece a la Oficina de Tutoría y Psicopedagogía de la Facultad de Educación de la Universidad Nacional Federico Villarreal de Lima, Perú.

\section{Fuentes de financiamiento:}

Recursos propios.

\section{Aspectos éticos / legales; Ethics / legals:}

Declaro haber actuado éticamente de acuerdo con las Conductas Responsables en Investigación.

\section{Conflicto de intereses:}

No se han presentado conflictos de intereses para el desarrollo del estudio, ni en la elaboración del presente manuscrito científico. 


\section{REFERENCIAS}

Abad, F., Garrido, J., Olea, J. y Ponsoda, V. (2006). Introducción a la Psicometría. Teoría Clásica de los Tests y Teoría de la Respuesta al Ítem. UAM

American Psychological Association (2017). Estilos de crianza. Disponible en: https:// www.apa.org/act/resources/espanol/crianza

Baker, F.B. (1992) Item response theory: Parameter estimation techniques. Marcel Dekker.

Bahls, S. C. (2002). Aspectos clínicos da depressão em crianças e adolescentes. Jornal de Pediatria, 78(5), 359-366

Beltrán, J. (1998). Procesos, estrategias y técnicas del aprendizaje. Síntesis

Bimbaum, A. (1968). Some latent trait models and their use in inferring an examinee's ability. En F.M. Lord y M.R. Novick, Statistical theories of mental test scores (p. 397-492). Reading, MA: Addison-Wesley.

Busko, D. (1998). Causes and consequences of perfectionism and procrastination: A structural equation model (Tesis de maestría). Guelph, University of Guelph

Castellá, J., Saforcada, E., Tonon, G., Rodríguez, L., Mozobancyk, S. y Bedin (2012). Bienestar subjetivo de los adolescentes: Un estudio comparativo entre Argentina y Brasil. Psychosocial Intervention, 21(3), 273-280. Disponible en: http://scielo.isciii. es/pdf/inter/v21n3/04.pdf

Caprara, G. V., Barbaranelli, C., Borgogni, L. \& Perugini, M. (1993). The Big Five Questionnaire: A new Questionnaire for the measurement of the Five factor model. Personality and Individual Differences, 15: 281-288

Castaños, C. y Sánchez, S., J. (2015). Niñas y adolescentes en riesgo de calle: Bienestar subjetivo y salud mental. Revista CES Psicología, 8(1), 120-133

Chao, T., Wang, Y. \& Sung, Y. (2012). Development of Scale of Examination Stress and Test Anxiety (SESTA) and the Examination Stress in Taiwan. Poster presentado en la 120th American Psychological Association Annual Convention

Chu, P., Saucier, D. \& Hafner, E. (2010). Meta-analysis of the relationships between social support and well-being in children and adolescents. Journal of Social and Clinical Psychology, 29, 624-645

Connor, K. M. \& Davidson, J. T. (2003). Development of a newresilience scale: The ConnorDavidson Resilience Scale (CD-RISC). Depression and Anxiety, 18(2), 76-82

Crawford, U. \& Manassis, K. (2011). Ansiedad, habilidades sociales, calidad de la amistad y victimización entre pares: un modelo integrado. Journal of Anxiety Disorders, 25(7): 924-931. https://doi.org/10.1016/j.janxdis.2011.05.005

Cuesta, M. (1996). Unidimensionalidad. En J. Muñiz (Ed.), Psicometría (pp. 239-292). Universitas

Díaz, L., Rosero, R., Melo, M. y Aponte, D. (2013). Habilidades para la vida: análisis de las propiedades psicométricas de un test creado para su medición. Revista Colombiana de Ciencias Sociales, 4(2), 181-200 
Diener, E. (1996). Traists can be powerful, but are not enough: lessons from Subjective Wellbeing. Journal of Research in Personality, 30, 389-399.

Diener, E., Oishi, S., \& Tay, L. (2018). Advances in subjective well-being research. Human Behavior, 2, 253-260. doi:10.1038/s41562-018-0307-6

Elosua, P., y Zumbo, B. (2008). Coeficientes de fiabilidad para escalas de respuesta categórica ordenada. Psicothema, 20, 896-901

Erceg-Hurn, D. M., y Mirosevich, V. M. (2008). Modern robust statistical methods: An easy way to maximize the accuracy and power of your research. American Psychologist, 63, 591-601. doi: 10.1037/0003-066X.63.7.591

Felder, R. y Silverman, L. (1988). Learning and Teaching Styles in Engineering Education. Engr. Education, 78(7), 674-681. Recuperado de http://www4.ncsu.edu/unity/ lockers/users/f/felder/public/Papers/LS-1988.pdf

Feitosa F, de Matos M, del Prette Z y del Prette A (2005). Suporte social, nível socioeconómico e o ajustamento social e escolar de adolescentes portugueses. Temas em Psicología, 13, 129-138

Felder, R. y Silverman, L. (1988). Learning and Teaching Styles in Engineering Education. Engr. Education, 78(7), 674-681. Recuperado de http://www4.ncsu.edu/unity/ lockers/users/f/felder/public/Papers/LS-1988.pdf

Fernández-Castillo, E., Camejo, Y., Rodríguez-Martín, B., Grau, J. y Grau, R. (2014). Análisis Factorial y Confiabilidad del autorreporte vivencial. Alternativas cubanas en psicología, 2(6), 85-91

Fernández, L. (2005). ¿Es posible conocer la personalidad? Problemas metodológicos para su estudio. Félix Varela

Fergus, S. \& Zimmerman, M. (2005). Adolescent resilience: Aframework for understanding healthy development in theface of risk. Annual Review of Public Health, 26, 399 419. Disponible en: http://dx.doi.org/10.1146/annurev.pubhealth.26.021304.144357

Ferrando, P.J., y Anguiano, C. (2010). El análisis factorial como técnica de investigación en Psicología. Papeles del Psicólogo, 31, 18-33

Fuentes, N., Medina, J., Van Barneveld, H. \& Escobar, S. (2012). Resiliencia y factores protectores en menores infractores y en situación de calle. Psicología y Salud, 22(1): $49-62$

Fraguela, J., Carral, L., Iglesias, G., Castro, A., y Rodriguez, M. J. (2011). La integración de los Sistemas de gestión. Necesidad de una nueva cultura empresarial. Open Journal Systems, 78(167): 44-49

García-Viniegras, C y González, I. (2000). La categoría bienestar psicológico. Su relación con otras categorías sociales. Rev. Cubana Med Gene Integr, 16(6), 586-592. Disponible en: http://scielo.sld.cu/pdf/mgi/v16n6/mgi10600.pdf

Gardner, H. (1993). Inteligencias múltiples. La teoría en la práctica. Paidós

Goss-Sampson, M. (2019). Análisis estadístico en JASP: una guía para estudiantes. 
Gutiérrez, M. y Gonçalves, T. (2013). Activos para el desarrollo, ajuste escolar y bienestar subjetivo de los adolescentes. International Journal of Psychology and Psychological Therapy, 13(3), 339-355. Disponible en: https://www.redalyc.org/articulo. oa? id $=560 / 56028282006$

Haladyna, T. M., y Rodríguez, M. C. (2013). Developing and validating test items. Routledge

Hudson, A. \& Nandy, K. (2012). Comparison of substance abuse, high-risk sexual behavior and depressive symptoms among homeless youth with and without a history of foster care placement. Contemp Nurse, 42(2), 178-186.

Hughes, J., Clark, S., Wood, W., Cakmak, S., Cox, A., MacInnis, M. \& Broom, B. (2010). Youth homelessness: the relationships among mental health, hope and service satisfaction. J Can Acad Child Adolesc Psychiatry, 19(4), 274-283.

Instituto Nacional de Estadística e informática (2018). Perú: indicadores de educación por departamento, 2007-2017. Lima: INEI. Recuperado de https://www.inei.gob.pe/ media/MenuRecursivo/publicaciones digitales/Est/Lib1529/libro.pdf

Karlsen, E., Dybdahl, R. \& Vitterso, J. (2006). The possible benefits of difficulty: how stress can increase and decrease subjective well-being. Scand J Psychol, 47, 411-417

Lemos, M.A. y Londoño, N. (2006). Construcción y Validación del Cuestionario de Dependencia Emocional en población colombiana. Acta Colombiana de Psicología, 9(2), 127-140. Disponible en: https://www.redalyc.org/pdf/798/79890212.pdf

Lent, R., Taveira, M., Sheu, H. y Singley, D. (2009). Social cognitive predictors of academic adjust Social cognitive predictors of academic adjustment and life satisfaction in Portuguese college students: A longitudinal analysis. Journal of Vocational Behavior, 74, 190-198

Meza, A. (2013). Estrategias de aprendizaje. Definiciones, clasificaciones e instrumentos de medición. Propósitos y Representaciones, 1(2), 193-213

Montero, I. y León, O. G. (2002). Clasificación y descripción de las metodologías de investigación en Psicología. Revista Internacional de Psicología Clínica y de la Salud, 2(3), 503-508

Muñiz, J. y Fonseca-Pedrero, E. (2019). Diez pasos para la construcción de un test. Psicothema, 31(1), 7-16. doi: 10.7334/psicothema2018.291

Muñiz, J. y Bartram, D. (2007). Improving international tests and testing. European Psychologist, 12, 206-219

Oliva, A., Ríos, M., Antolín, L., Parra, A., Hernando, A. y Pertegal, M. A. (2010). Más allá del déficit: Construyendo un modelo de desarrollo positivo adolescente. Infancia y Aprendizaje, 33, 223-234

Olivera, M. y Simkin, H. (2016). Factores de la personalidad y su relación con el bienestar subjetivo y psicológico. Hologramática, 6, 77-96

Palummo, J. (2013). La situación de niños, niñas y adolescentes en las instituciones de protección y cuidado de América Latina y el Caribe. UNICEF 
Pavot, W. \& Diener, E. (2013). Happiness experienced: The scienceof subjective wellbeing. En S. A. David, I. Boniwell, \& A. Conley Ayers (Eds.), The Oxford Handbook of Happiness (pp. 134-151). Oxford University Press

Peñate, W., Bello, R., García, A., Rovella, A. y Del Pino-Sedeño, T. (2014). Características psicométricas del Cuestionario Básico de Depresión en una muestra de adolescentes. Anales de psicología, 30(1), 143-149

Pertegal, M., Oliva, A. y Hernando, A. (2010). Los programas escolares como promotores del desarrollo positivo adolescente. Cultura y Educación, 22, 53-66

Rodríguez-Fernández, A., Ramos-Díaz, E., Ros, I., Fernández-Zabala, A. y Revuelta, L. (2016). Bienestar subjetivo en la adolescencia: el papel de la resiliencia, el autoconcepto y el apoyo social percibido. Suma psicológica, 23(1): 60-69. https://doi. org/10.1016/j.sumpsi.2016.02.002

Sánchez-Villena, A. (2019). Uso de programas estadísticos libres para el análisis de datos: Jamovi, Jasp y R. Revista Perspectiva, 20(1), 112-114.

Skin, D. \& Johnson, R. (1978). Avowed happiness as an overall assessment of quality of life. Soc Indicat Res, 5, 475-492

Spielberger, C. (1966). Theory and research on anxiety. En C.D. Spielberger (Ed.), Anxiety and Behavior (p. 3-22). Academic Press

Tenorio, M. (2011). Adolescencia, pareja y violencia familiar. Temática Psicológica, 7(1): 25-32.

Trujillo-Chumán, K. y Noé-Grijalva, M. (2020). La escala de Procrastinación académicaEPA: validez y confiabilidad en una muestra de estudiantes peruanos. Revista de Psicología y Educación, 15(1), 98-107. doi: https://doi.org/10.23923/rpye2020.01.189

Ugarriza, N., y Escurra, M. (2002). Adaptación psicométrica de la Escala de Depresión para Adolescentes de Reynolds (EDAR) en estudiantes de secundaria de Lima Metropolitana. Persona, 5, 83-130

Verdugo-Lucero, J. L., Ponce de León-Pagaza, B. G., Guardado-Llamas, R. E., MedaLara, R. M., Uribe-Alvarado, J. I., \& Guzmán-Muñiz, J. (2013). Estilos de afrontamiento al estrés y bienestar subjetivo en adolescentes y jóvenes. Revista Latinoamericana de Ciencias Sociales, Niñez y Juventud, 11(1), 79-91. doi:10.11600/169271 $5 x .1114120312$

Wright, M., Masten, A. S. \& Narayan, A. J. (2013). Resilience processes in development: Four waves of research on positiveadaptation in the context of adversity. En S. Goldstein, \& R. B.Brooks (Eds.), Handbook of Resilience in Children (pp. 15-37). Springer Science

Yulianti, L., Ramalis, T. R., \& Purwanto, P. (2020). Karakteristik Tes Keterampilan Berpikir Kritis Pada Materi Hukum Newton Berdasarkan Teori Respon Butir. WaPFi (Wahana Pendidikan Fisika), 5(1), 66-70. http://libirt.psychometricon.net/releases/

Zimet G, Dahlem, Zimet S, Farley G. The multidimensional scale of perceived social support. Journal of Personal Assessment. 52(1): 30-41. 